\title{
MAGNETIC RESONANCE IMAGING 3T AND TOTAL FIBROTIC VOLUME IN AUTOSOMAL DOMINANT POLYCYSTIC KIDNEY DISEASE
}

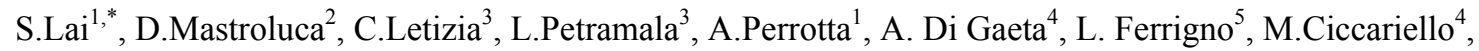 \\ A.R.D'Angelo ${ }^{6}$, V.Panebianco ${ }^{4}$.
}

\author{
${ }^{1}$ Department of Clinical Medicine, Sapienza University of Rome, Rome, Italy \\ ${ }^{2}$ Nephrology and Dialysis Unit, Hospital ICOT Latina, Sapienza University of Rome, Italy \\ ${ }^{3}$ Department of Internal Medicine and Medical Specialities, Sapienza University of Rome, Rome, \\ ${ }^{4}$ Department of Radiological, Oncological and Pathological Sciences, Sapienza University of Rome, Italy \\ ${ }^{5}$ National Centre for Epidemiology, Surveillance and Health Promotion, National Institute of Health, Rome \\ ${ }^{6}$ Department of Obstetrical-Gynecological Sciences and Urologic Sciences, Sapienza University of Rome, Italy
}

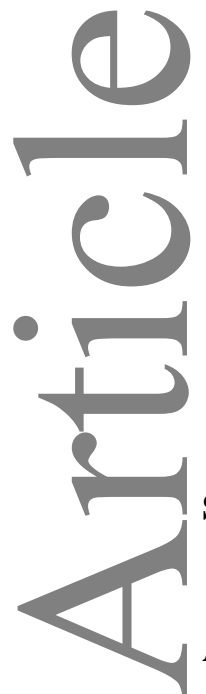

Corresponding author:

Silvia Lai, MD

Assistant Professor of Nephrology

Department of Clinical Medicine

Sapienza University of Rome

Viale dell'Università 3700185 Rome, Italy

Telephone number: +39393384094031

Fax number +390649972068

E-mail address: silvia.lai@uniroma1.it

Short title: MARKERS OF PROGRESSION in ADPKD

\section{ABSTRACT}

INTRODUCTION Autosomal dominant polycystic kidney disease (ADPKD) is the most common renal hereditary disorder. Several authors have attempted to identify a kidney damage marker for predicting the prognosis and the effectiveness of therapy in ADPKD. The aim of this study was to identify and quantify in ADPKD, through a novel MR protocol with 3 Tesla (MRI 3Tesla), the presence of parenchymal fibrotic tissue at early stage of disease, able to correlate the glomerular filtrate and to predict the loss of the function renal.

MATERIAL AND METHODS 15 ADPKD patients undergone to renal MRI 3Tesla at T0 and revaluated after follow up (T1) of 5 years. We have evaluated renal function, plasma aldosterone concentration (PAC), insulin resistance and surrogate markers of atherosclerosis (carotid intima media thickness (IMT), ankle/brachial index (ABI) and left ventricular mass index (LVMI).

RESULTS Our study showed a significant negative correlation between total kidney volume and estimated glomerular filtration rate $(\mathrm{eGFR})$ during observational observation $(\mathrm{p}<0.02)$. Moreover, we showed a negative correlation between eGFR with Total Fibrotic Volume $($ TFV) $(\mathrm{p}<0.04)$ and Total Perfusion Volume/Total kidney Volume $(<0.02)$. Moreover TFV was correlated positively with PAC $(p<0.05)$, insulin values $(p<0.05)$, ABI $(p<0.05)$ and $\operatorname{LVMI}(\mathrm{p}<0.01)$.

CONCLUSIONS The MRI 3Tesla, despite the high costs, could be considered an useful and non-invasive method in the evaluation of fibrotic tissue and progression of the disease in ADPKD patients. Further clinical trials on larger group are due to confirm the results of this pilot study, suggesting that MRI 3Tesla can be useful to evaluate the effectiveness of new therapeutic strategies.

Keywords: Autosomal dominant polycystic kidney disease, total volume fibrotic, plasma aldosterone, subclinical atherosclerosis parameters.

This article has been accepted for publication and undergone full peer review but has not been through the copyediting, typesetting, pagination and proofreading process, which may lead to differences between this version and the Version of Record. Please cite this article as doi: 10.1111/imj.14039 


\section{INTRODUCTION}

Autosomal dominant polycystic kidney disease (ADPKD) is most common kidney genetic disease with a prevalence from 1:400 to 1:1000 live births, characterized by multiple and bilateral cystic dilatation of the renal tubules that lead to an enlargement in the total kidney volume (TKV) and to kidney failure. ADPKD can arise from mutations in either the PKD1 gene (cr. 16p13.3), which encodes the protein polycystin 1 or the PKD2 gene (cr. 4p13q23), encoding polycystin 2 (1). PKD1-associated disease presents a more severe prognosis and an earlier progression to end-stage renal disease (ESRD) respect to PKD2, with the mean age of 54 years for PKD1 and 74 years for PKD2 to developing renal insufficiency (2). The renal function remain normal for many years with an inexorably rapid decline in the final stage of disease in ADPKD patients. Caroli et al (3) showed that in an advanced stage of disease, the main component of the damage is the tubule interstitial fibrosis. Fibroblasts and collagen fibers build up in the parenchyma with different distribution: collagen type I and III are located in the interstice, while collagen type IV is in the basement membrane. Interstitial fibrosis could come from cortical ischemia produced by the vascular lesions and luminal narrowing (4-5).

The tubule-interstitial damage could also be caused by cytokines and chemokines released from inflammatory cells pressent in kidney tissue; this is also supported by large infiltrated of macrophages in the fibrotic tissue. Another important role in the pro-inflammatory factors secretion could be played by aldosterone. High levels of aldosterone enhance the expression of osteopontina, MCP-1, IL-6 and IL-1 $\beta$. Aldosterone has also direct proliferative and fibrogenetic activity respectively mediated by its functions on MAPK in the nucleus and its ability to increase TGF $\beta$ production (6-7). All these mechanisms are very important because currently, we have the need of novel biomarkers of functional loss and indexes of disease progression, especially for testing new therapeutic strategies. The evaluation of fibrotic progression in ADPKD kidneys, estimated with specific Magnetic Resonance Imaging (MRI) sequences, could be a promising predictive factor for the evaluation of the onset of renal damage (8).

\section{AIM OF THE STUDY}


The aim of study was to identify and quantify the parenchymal fibrotic tissue, possible marker of kidney damage at an early stage of the disease with MRI, correlating with the estimated glomerular filtration rate (eGFR), predicting the loss of kidney function.

\section{MATERIALS AND METHODS}

The study protocol was approved by the Local Clinical Research Ethics Committee with protocol number 3169/15. The study conforms to the principles outlined in the Declaration of Helsinki and we obtained a written consent by each patient enrolled.

\section{Study design and subjects}

We performed a prospective longitudinal study on ADPKD patients at the University Hospital "Policlinico Umberto I" of Rome, Sapienza University of Rome, Italy. Patients were consecutively enrolled from July 2010 and June 2016. Clinical, laboratory, instrumental parameters were evaluated at baseline (T0) and at 5 years (T1).

\section{Patients}

15 ADPKD patients (4 males and 11 females, mean age 39.0 99.2 years) were evaluated at time of diagnosis (T0) and after follow-up (T1). The eGFR was evaluated according to the modification of diet in renal disease formula (9), CKDEpidemiology and creatinine clearance in a 24-hour urine. We enrolled patients both normotensive and hypertensive. The state of arterial hypertension was defined by use of hypotensive drugs (angiotensin converting enzyme (ACEIs), Angiotensin II receptor blockers (ARBs), beta-blockers, calcium antagonists, alpha-lytic and / or diuretics) or by the presence of a pressure higher than $140 / 80 \mathrm{~mm} \mathrm{Hg}$ in three consecutive measurements. Patients on antihypertensive treatment with ACEIs and/or ARBs, beta-blockers and alpha-lytic have been subjected to a wash-out period of 15 days by replacing the hypotensive therapy with clonidine.

\section{Inclusion criteria}

Patients aged $>18$ years with diagnosis of ADPKD, defined according to the Pei's criteria (10).

\section{Exclusion criteria}

We excluded patients having kidney surgery, or submitted to the cyst drainage procedures, or patients with other diseases that can potentially affect kidney function, patients with metal clip carriers, or patients in pregnant or nursing patients. Moreover, patients that refused to give consent and patients with missing data were also excluded.

\section{Laboratory measurements}


Right and left carotid ultrasound was blindly performed by an experienced sonographer (S.L.) who was unaware of the characteristics of the patients under examination. Participants were studied with the high-resolution B-mode ultrasound machine, Toshiba Aplio XV (Toshiba Aplio XV, Toshiba American Medical Systems, Inc., Tustin, CA, USA) equipped with a 5- to $12-\mathrm{MHz}$ linear transducer with a $0.01-\mathrm{mm}$ resolution, following a standardized vascular protocol. IMT was measured at three points on the far walls of both left and right distal common carotid arteries, carotid bulb, and the proximal portion of the internal carotid arteries (16). The mean IMT was computed as the average IMT on both sides. The value of IMT was considered normal when between 0.55 and $0.9 \mathrm{~mm}$ (17).

\section{Magnetic Resonance Imaging (MRI)}

All patients were subjected to a novel MRI protocol of advanced imaging with magnet 3T (Discovery MR 750, 3T, GE Healthcare) after positioning of the surface coil 32 channels. The acquisition protocol included morphological sequences, single shot T2-weighted (SSFS)(TR 850 ms, TE 105 ms; Flip Angle 90; FoV 320x320; Matrix 320x224) acquired on axial, sagittal and coronal planes and sequences Gradient Echo (GRE ) T1-weighted (TR 5 ms; TE 1ms; Flip Angle 15; FoV 420x420; 288x192 matrix). For the evaluation of parenchymal perfusion were used sequences ultrafast GRE T1-weighted, acquired in the coronal plane (TR $2 \mathrm{mS}$; TE $1 \mathrm{mS}$; Flip Angle 13; Thickness $200 \mathrm{~mm}$; FoV 300x300 mm, matrix 192x138) during administration dynamic of i.v. contrast (Gadobutrol $1 \mathrm{mmol} / \mathrm{ml}$, Gadovist, Bayern) using a perfusion technique, with high temporal resolution of 4 seconds, for a total duration of about 8 minutes. The departure of the dynamic sequence was made to coincide with the start of the administration of contrast material i.v. (18). The evaluation of Total Perfusion Volume (TPV) and Total Fibrotic Volume (TFV) results from Perfusional MRI after a qualitative and quantitative approach. Each parameter resulted from a post-processed slice by slice renal segmentation rispectively in early arterial phase (1st minute of perfusion) and late perfusional fase (8th minute of perfusion). Segmentation was guided using colorimetric maps. After segmentation, software Workstation vers. 4.6 was

used for 3D volume rendering reconstrucion, which resulted in semiquantitative estimation of parenchymal perfonded tissue and fibrotical areas. These perfusional parameters give indication of functional parenchimal areas. Perfusional volume indicates kidney's areas where blood flow is preserved, which is an indirect sign of normal functional parenchyma. Fibrotical areas indicate parenchymal areas which underwent to fibrotical substitution and consequent loss of function.

\section{Image analysis}

\section{Morphological Assessment}

In the morphological images HASTE T2 and T1-weighted 3D GRE, the following parameters were evaluated: kidney size on 3 floors of acquisition (axial sagittal and coronal), cortical thickness, cortico-medullary differentiation, volume of cysts. In the 3D T1 weighted GRE sequences, the morphological characteristics and the signal intensity, relating to 
the presence or absence of a high protein content have been evaluated, allowing the identification of hemorrhagic cyst content, infected cysts and degenerate cysts.

\section{Qualitative and Quantitative Assessment}

The perfusion technique was evaluated with both qualitative and quantitative approach. In the first case, two types of colorimetric maps were obtained, reconstructed both at an early stage that at a late stage. The colorimetric map elaborated in the early stage reflects an enhancement of arterial type, derived from the analysis of the early stages, which identify the normally perfused renal parenchyma, and which may be defined as "functional." The colorimetric map developed in the late phase, shows the strengthening of focal renal parenchymal areas, that may identify areas of parenchymal fibrosis. The colorimetric maps also allow a 3D three-dimensional reconstruction of the kidney (Volume Rendering achieved by GE, Workstation software vers. 4.6) to highlight the renal parenchyma perfused, called "perfusion volume". This result was obtained by using the colorimetric map derived from early-stage acquisitions that corresponds to approximately 35 seconds after the beginning of injection of the contrast medium and the colorimetric maps obtained in the late phase, which corresponds to about 8 minutes from the start of injection of the contrast agent; the latter made it is possible to evaluate the estimate of parenchymal fibrosis defined as "fibrotic volume" (19).

\section{Statistical analysis}

Data management and analysis were performed using IBM ${ }^{\circledR}$ SPSS ${ }^{\circledR}$ Statistics 22.0 for Windows ${ }^{\circledR}$ software (IBM Corporation, New Orchard Road Armonk, New York, United States). The normality of variables was tested using the Shapiro-Wilk method for normal distributions. All continuous variables were expressed as mean \pm standard deviation, categorical variables were expressed as number (percentage). Spearman's correlation was used to determine in bivariate correlation the relationship and the strength of association between the variables. A probability value of $p<0.05$ was considered to be statistically significant.

\section{RESULTS}

The clinical charasterics of ADPKD patients are shown in Table 1. The mean follow-up was $52.80 \pm 17.95$ months. As awaited, renal function has been gradually reduced in all patients. The slope of eGFR varies from -0.06 to -0.56 $\mathrm{mL} / \mathrm{mi} / 1.73 \mathrm{~m}^{2} / \mathrm{month}$. The assessment of renal diameters in coronal, axial and sagittal allowed the calculation of the height-adjusted total kidney volume (htTKV) (Table 1). MRI showed by colorimetric maps obtained both in early and late phase of the parenchymal fibrosis share, defined as "fibrotic volume" (Figure 1) and proportion of perfused parenchyma, defined as "perfusion" volume (Figure 2), it was $330.57 \pm 266.10$ and $392.40 \pm 402.83 \mathrm{~cm}^{3}$, respectively

(Table 1). The mean value of htTKV was result $840.08 \pm 816.72 \mathrm{~cm}^{3}$. The mean ratio between the total perfusion volume and total kidney volume, expressed as a percentage (TPV/TKV\%) varies from $22 \%$ to $107 \%$ at $\mathrm{T} 0$ and from 
$12 \%$ to $91 \%$ at follow up (T1). The analysis of correlations between the TKV and eGFR at follow up (T1), showed a statistically significant negative correlation between the two parameters $(r=-0.59, p=0.02)$, while the same parameters evaluated at T0 showed no correlation (Figure 3). We found a trend of correlation between the TPV and eGFR at T1 $(\mathrm{r}=-12.48, \mathrm{p}=0.06)$. Moreover, we found a negative correlation between eGFR and TFV $(\mathrm{r}=-0.61, \mathrm{p}=0.04)$, and eGFR with TPV/TKV \% $(r=-0.59, \mathrm{p}=0.02)$ at T1 (Figure 4). No significative correlations were found between TKV (r=0.37, $\mathrm{p}=0.16)$ and TPV/TKV \% $(r=-0.16, \mathrm{p}=0.56)$ with progressive reduction of eGFR. Moreover, in this study PAC was correlated positively with the TFV $(\mathrm{r}=0.50, \mathrm{p}=0.05)$. Subdividing patients in according to age (21-34 and 35-51 years), aldosterone showed a significant correlation with the higher age class $(r=0.88, p<0.003)$, and not in lower age ( $r=-$ $0.03, \mathrm{p}=0.93)$. Our study also showed a positive correlation between the TFV with insulin values $(\mathrm{r}=0: 49, \mathrm{p}<0.05), \mathrm{ABI}$ index $(\mathrm{r}=0.51, \mathrm{p}<0.05)$ and LVMI $(\mathrm{r}=0.63, \mathrm{p}<0.01)$, but no significant correlation with IMT. PRA was in the normal range in all patients (data not shown).

\section{DISCUSSION}

In the present study, we investigated a novel MRI protocol, using advanced imaging with 3Tesla high field magnet, that through colorimetric Maps in the early and late phase, with 3D three-dimensional reconstruction, identifies areas of parenchyma perfused called "perfusion volume", and areas of renal fibrosis defined "fibrotic volume". Total Fibrotic Volume is a noncystic area of the polycystic kidney, characterized by peritubular interstitial fibrosis, tubular dilation, atrophy and vascular sclerosis. Total Perfusion Volume represents the noncystic fraction of the polycystic kidney with a normal perfusion and tissue architecture. It is known that eGFR is a late marker of kidney damage in ADPKD, while the presence of fibrosis was documented in an early phase of disease, with preserved renal function and TKV slightly increased. The sequential evaluation of TKV and the cystic volume has been adopted in recent years in several clinical trials as a measure of outcome, but these showed no linear correlation with worsening renal function, if not in the late stages of the disease. In fact, the Consortium for Radiologic Imaging in Polycystic Kidney Disease cohort (CRISP) study, showed that in ADPKD patients Magnetic resonance-based htTKV over $600 \mathrm{ml} / \mathrm{m}$ predicts the development of CKD Stage 3 within 8 years, and that TKV and cyst volume increase exponentially with age, but causal relationship between kidney or cyst volume increase and the progression to ESRD has not been completely demonstrated. In our study, TKV was negatively correlated with eGFR at T1, in according with CRISP study (8), while the same parameters evaluated at T0 didn't showed any correlation, confirming that TKV and eGFR could not be correlated in the early stages of disease. Moreover, we showed a negative correlation between eGFR and TFV, and a positive correlation between eGFR and TPV/TKV \% at T1, suggesting these as possible new markers that could be used to assess disease progression and efficacy to therapy. Instead, TPV showed a negative correlation, albeit without statistical significance, with eGFR at T1, perhaps this may also be due to the low number of the sample examined. Caroli et al.(3) have 
characterized extracystic tissue on contrast-enhanced computed tomography (CT), subdividing extracystic tissue into two separate components, fully enhanced and hypoenhanced parenchyma tissue, called "intermediate", and they showed that intermediate tissue significantly correlated with eGFR. Moreover in our study we showed that PAC was correlated positively with the TFV, and subdividing patients into age groups (21-34 and 35-51 years), PAC showed a significant correlation with the higher age class, resulting however not correlated in patients with a lower age. The results obtained could confirm an involvement of the aldosterone in the development of renal fibrosis, especially in advanced stages, the result of a mechanism which starts already in the initial stage of the disease, and that gradually leads to irreversible loss of renal parenchyma. On the contrary, the PRA was normal in all patients. Valvo and Other authors (20) have suggested that the suppression of the renin-angiotensin-aldosterone and low renin values in ADPKD patients could be due to an increased plasma volume. The production of aldosterone, has been demonstrated also in extra adrenal tissue and appears (2) to be only partially influenced by the plasma levels of angiotensin II (21). It would be connected to the production of extracellular matrix and could, therefore, have a pathophysiological role in the genesis and progression of chronic kidney damage. In addition, normal PRA, does not exclude the presence of a strongly activated intrarenal RAAS, demonstrated in ADPKD patients (22), with a local overproduction of angiotensin II and aldosterone, and consequent hemodynamic and chronic inflammatory tissue action $(7,23)$. The hyperproduction of aldosterone determines arteriolar vasoconstriction, production of cytokines and adhesion molecules with consequent recall of inflammation cells, chemotaxis, activation of macrophages and growth of fibroblasts (5). It is able, moreover, to stimulate coagulation, through the increase plasminogen activator inhibitor type I, the activation of platelets and the increase of their aggregation at the level of bleeding sites. Increased levels of aldosterone and angiotensin II could lead to systemic fibrosis, mitogenesis and stimulate the formation of cysts (6). Many studies support the evidence of aldosterone role in determining a cardiovascular damage, regardless of angiotensin II and the blood pressure (5-6,11). Indeed the hormone could have a fibrotic effect on vascular smooth muscle cells, endothelial cells, cardiomyocytes and renal tissue (24). Our results showed that TFV, was correlated positively also with insulin values, ABI index and LVMI. Currently, the link between the function of polycystins and insulin value, is still little known, although recently Mao et al. (25) have shown that polycystins can regulate the secretion of insulin, being expressed also in the beta cells of pancreatic islet. ABI is a simple, non-expensive and non-invasive marker of atherosclerosis, in fact had a high specificity to predict future cardiovascular outcomes. Price et al. (13) showed that the combination of IMT and ABI, could have an additive effect for predicting CVD, also if in our study only ABI and not IMT resulted correlate with TFV. However, Tokiwa et al. (26) showed that in ADPKD patients with preserved renal function, ABI found no significant relationship with disease progression, while in our study ABI was significantly correlate with TFV. Currently, we have the need of novel biomarkers of functional loss, indexes of disease progression, especially to monitor the effectiveness of therapy. In fact 
the relationship between eGFR and TKV is stronger during the later stages of disease and total cyst and kidney volume, have been often utilized as outcome measures in clinical trials, but Caroli et al. (3) showed that intermediate volume, as determined by CT, comparing with gold standard (three ADPKD patients underwent bilateral nephrectomy), mainly constituted by tubule interstitial fibrosis, would be considered a better candidate marker for monitoring disease progression and, as outcome measures in ADPKD patients. Renal biopsy could represent the gold standard procedure but it could be negative and harmful, with possibility to aggravate renal function, especially considering the high rates of complications following kidney biopsy. Already in 1960 Landmark et al. showed that renal fibrosis is the best histological correlate of the progression of renal damage and long-term prognosis. The decline in renal function in ADPKD patients remain underhand for years, as a consequence of increased GFR by surviving nephrons. The compression of expanding cysts on parenchymal tissue and the stretching of renal microvessels cause hypoperfusion (2) and disruption of the normal tissue architecture leading to progressive renal functional loss. Tubulointerstitial damage, is now recognized as an essential element in the decline of renal function. Recent findings that intermediate volume, as determined by CT (3), is mainly constituted by interstitial fibrosis, confirm that this imaging parameter could be considered as a strong candidate marker for monitoring progression of renal disease, outcomes and therapy efficacy in ADPKD patients. 3 Tesla-Diffusion Tensor Imaging without contrast agent is already been used in ADPKD patients by Lupica et al (27), with promising results on the evaluation of the fractional anisotropy and apparent diffusion coefficient and a good correlation with kidney function tests, but this method without contrast agent, is certainly safer with low eGFR $(<30 \mathrm{ml} / \mathrm{min})$, but is not able to assess renal fibrous component. Therefore quantification and monitoring of fibrotic tissue and its evaluation relative to the preserved parenchyma could allow to stratify ADPKD patients, monitor disease progression and evaluate the effects of early treatment strategies when renal function is still preserved and patients are considered not at high risk cause to normal eGFR.

Moreover, renal biopsy could represent the gold standard procedure but it could be negative and harmful, with possibility to aggravate renal function, especially considering the high rates of complications following kidney biopsy (rupture of cysts, infection and even greater difficulties at the time of transplantation).

\section{LIMITATION OF THE STUDY}

This study is conducted in small cohort of ADPKD patients, therefore, it should be considered as hypothesis generating data, which need to be confirmed by further clinical studies with a larger number of patients. Moreover, the technique used to quantify the fibrotic and perfusion volume is "time consuming", and can be considered limited to specialized centers with advanced imaging techniques. Therefore, a large clinical trials is necessary to confirm our findings results and that further long-term data on larger population is needed to recommend the procedure in the clinical practice. 


\section{CONCLUSION}

Our results showed a possible method of advanced imaging, capable of identifying and quantifying the fibrotic and perfusion renal volume, already at an early stage of the disease, also with preserved renal function and with a possible correlation with the eGFR. Indeed novel MRI protocol of advanced imaging with 3Tesla high field magnet could accurately reproduce these changes, even when renal function is still preserved. If our hypothesis is confirmed by larger clinical studies, it would be advisable to carry out a study with MRI 3Tesla high field magnet, in the follow up of ADPKD patients, to evaluate the progression of the disease and the effectiveness of new therapeutic strategies, despite the high costs.

\section{"Compliance with Ethical Standards"}

The authors alone are responsible for the content and writing of the paper.

The manuscript has been seen and approved by all authors.

This study was not funded.

The manuscript is not under consideration for publication elsewhere.

The authors report no conflicts of interest.

The study protocol was in accordance with the ethical standards of the Local Clinical Research Ethics Committee at which the studies were conducted (IRB approval number 3169/15) and with the 1964 Helsinki declaration and its later amendments or comparable ethical standards.

Informed consent was obtained from all individual participants included in the study.

\section{(1) REFERENCES}

1) Torres VE, Harris PC, Pirson Y. Autosomal dominant polycystic kidney disease. Lancet 2007;369:1287-1301.

2) Hateboer N., v Dijk M.A., Bogdanova N., Coto E., Saggar-Malik A.K., San Millan J.L., Torra R., Breuning M., Ravine D. Comparison of phenotypes of polycystic kidney disease types 1 and 2: European PKD1-PKD2 Study Group. Lancet. 1999;353:103-107.

3) Caroli A, Antiga L, Conti S, Sonzogni A, Fasolini G, Ondei P, Perico N, Remuzzi G, Remuzzi A. Intermediate volume on computed tomography imaging defines a fibrotic compartment that predicts glomerular filtration rate decline in autosomal dominant polycystic kidney disease patients. Am J Pathol. 2011 Aug;179(2):619-27.

4) Mangos S, Lam P-Y, Zhao A, Liu Y, Mudumana S, Vasilyev A, Liu A, Drummond IA. The ADPKD genes pkd1a/b and pkd2 regulate extracellular matrix formation. Dis. Models \& Mech. 2010; 3:354-365.

5) Jill Norman: Fibrosis and progression of Autosomal Dominant Polycystic Kidney Disease (ADPKD): Biochim Biophys Acta. 2011 October; 1812(10): 1327-1336.

6) Liu Y. Renal fibrosis: New insights into pathogenesis and therapeutics. Kidney Int. 2006; 69:213-217.

7) Nakamura T, Ushiyama C, Suzuki S, Ebihara I, Shimada N, Koide H. Elevation of serum levels of metalloproteinase-1, tissue inhibitor of metalloproteinase-1 and type IV collagen, and plasma levels of metalloproteinase-9 in polycystic kidney disease. Am. J. Nephrol. 2000; 20:32-36.

8) Irazabal MV, Rangel LJ, Bergstralh EJ, Osborn SL, Harmon AJ, Sundsbak JL, Bae KT, Chapman AB, Grantham JJ, Mrug M, Hogan MC, El-Zoghby ZM, Harris PC, Erickson BJ, King BF, Torres VE8; CRISP Investigators. Imaging classification of autosomal dominant polycystic kidney disease: a simple model for selecting patients for clinical trials. J Am Soc Nephrol. 2015 Jan;26(1):160-72. 
9) Levey AS, Coresh J, Greene T, Stevens LA, Zhang YL, Hendriksen S, Kusek JW, Van Lente F; Chronic Kidney Disease Epidemiology Collaboration. Using standardized serum creatinine values in the modification of diet in renal disease study equation for estimating glomerular filtration rate. Ann Intern Med. 2006 Aug 15;145(4):247-54.

10) Pei Y, Obaji J, Dupuis A, Paterson AD, Magistroni R, Dicks E, Parfrey P, Cramer B, Coto E, Torra R, San Millan JL, Gibson R, Breuning M, Peters D, Ravine D. Unified criteria for ultrasonographic diagnosis of ADPKD. J Am Soc Nephrol. 2009 Jan;20(1):205-12.

11) Lai S, Petramala L, Mastroluca D, Petraglia E, Di Gaeta A, Indino E, Panebianco V, Ciccariello M, Shahabadi HH, Galani A, Letizia C, D'Angelo AR.Hyperaldosteronism and cardiovascular risk in patients with autosomal dominant polycystic kidney disease. Medicine (Baltimore). 2016 Jul;95(29):e4175.

12) Williams B, Poulter NR, Brown MJ, et al. BHS guidelines working party, for the British Hypertension Society. British Hypertension Society guidelines for hypertension management 2004 (BHS-IV): summary. BMJ. 2004 Mar $13 ; 328(7440): 634-40$.

13) Price JF, Tzoulaki I, Lee AJ, Fowkes FG. Ankle brachial index and intima media thickness predict cardiovascular events similarly and increased prediction when combined. J Clin Epidemiol. 2007 Oct;60(10):1067-75. Epub 2007 Jul 25.

14) Lang RM, Bierig M, Devereux RB, et al. Chamber Quantification Writing Group; American Society of Echocardiography's Guidelines and Standards Committee; European Association of Echocardiography. Recommendations for chamber quantification: a report from the American Society of Echocardiography's Guidelines and Standards Committee and the Chamber Quantification Writing Group, developed in conjunction with the European Association of Echocardiography, a branch of the European Society of Cardiology. J Am Soc Echocardiogr. 2005;18(12):1440-1463

15) Lai S, Coppola B, Dimko M, et al. Vitamin D deficiency, insulin resistance, and ventricular hypertrophy in the early stages of chronic kidney disease. Ren Fail. 2014 Feb;36(1):58-64.

16) Ho CY, Solomon SD. A clinician's guide to tissue Doppler imaging. Circulation. 2006; 113(10): 396-398.

17) Lai S, Mariotti A, Coppola B, et al. Uricemia and homocysteinemia: nontraditional risk factors in the early stages of chronic kidney disease--preliminary data. Eur Rev Med Pharmacol Sci. 2014;18(7):1010-7.

18) Panebianco V, Barchetti F, Musio D, Forte V, Pace A, De Felice F, Barchetti G, Tombolini V, Catalano C.Metabolic atrophy and 3-T 1H-magnetic resonance spectroscopy correlation after radiation therapy for prostate cancer. BJU Int. 2014 Dec;114(6):852-9.

19) Michaely HJ, Kramer H, Attenberger U, Sourbron SP, Weckbach S, Reiser MF, Schoenberg SO. Renal magnetic resonance angiography at $3.0 \mathrm{~T}$ : technical feasibility and clinical perspectives. Top Magn Reson Imaging. 2007 Apr;18(2):117-25.

20) Valvo E, Gammaro L, Tessitore N, Panzetta G, Lupo A, Loschiavo C, Oldrizzi L, Fabris A, Rugiu C, Ortalda V, Maschio G: Hypertension of polycystic kidney disease: Mechanisms and hemodynamic alterations. Am J Nephrol 2001; $5: 176-181$.

21) Loghman-Adham M, Soto CE, Inagami T, Cassis L.: The intrarenal renin-angiotensin system in autosomal dominant polycystic kidney disease. Am J Physiol Renal Physiol. 2004 Oct;287(4):F775-88.

22) Harrap SB, Davies DL, Macnicol AM, Dominiczak AF, Fraser R, Wright AF, Watson ML, Briggs JD: Renal, cardiovascular and hormonal characteristics of young adults with autosomal dominant polycystic kidney disease. 1991; Kidney Int 40: 501-508.

23) Antiga L, Piccinelli M, Fasolini G, Ene-Iordache B, Ondei P, Bruno S, Remuzzi G, Remuzzi A. Computed tomography evaluation of autosomal dominant polycystic kidney disease progression: a progress report. Clin J Am Soc Nephrol. 2006 Jul;1(4):754-60. 
24) Bhutani H, Smith V, Rahbari-Oskoui F, Mittal A, Grantham JJ, Torres VE, Mrug M, Bae KT, Wu Z, Ge Y, Landslittel D, Gibbs P, O'Neill WC, Chapman AB1; CRISP Investigators. A comparison of ultrasound and magnetic resonance imaging shows that kidney length predicts chronic kidney disease in autosomal dominant polycystic kidney disease. Kidney Int. 2015 Jul;88(1):146-51.

25) Mao Z, Xie G, Ong AC.Metabolic abnormalities in autosomal dominant polycystic kidney disease. Nephrol Dial Transplant. 2015 Feb;30(2):197-203.

26) Tokiwa S, Muto S, China T, Horie S.The relationship between renal volume and renal function in autosomal dominant polycystic kidney disease. Clin Exp Nephrol. 2011 Aug;15(4):539-45.

27) Lupica R, Mormina E, Lacquaniti A, Trimboli D, Bianchimano B, Marino S, Bramanti P, Longo M, Buemi M, Granata F. 3 Tesla-Diffusion Tensor Imaging in Autosomal Dominant Polycystic Kidney Disease: The Nephrologist's Point of View.. Nephron. 2016;134(2):73-80. Epub 2016 Aug 10.
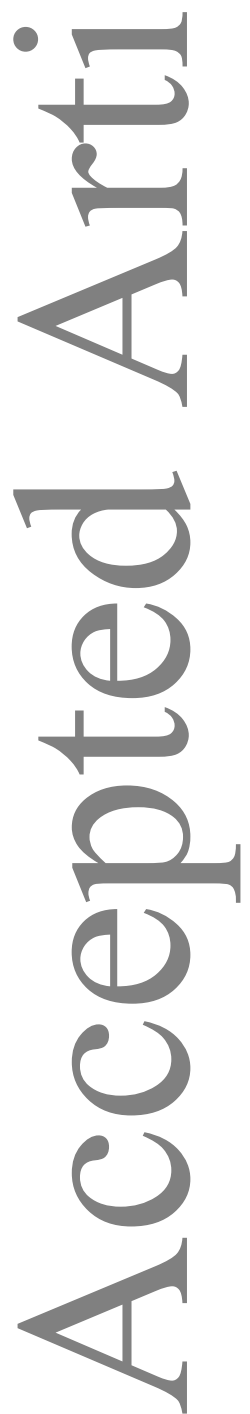


\begin{tabular}{|c|c|c|c|c|c|c|c|c|c|c|}
\hline $\begin{array}{l}\text { ADPKD } \\
\text { Patiens }\end{array}$ & Age & $\begin{array}{c}\text { eGFR } \\
\text { T0 }\end{array}$ & $\begin{array}{c}\text { PAC } \\
\mathrm{pg} / \mathrm{mL}\end{array}$ & $\begin{array}{c}\text { TFV T1 } \\
\mathrm{cm}^{3}\end{array}$ & $\begin{array}{c}\text { TPV/TKV } \\
\text { (\%) T0 }\end{array}$ & $\begin{array}{c}\text { TPV/TKV } \\
\text { (\%) T1 }\end{array}$ & $\begin{array}{c}\text { TKV TO } \\
\mathrm{cm}^{3}\end{array}$ & $\begin{array}{c}\text { TKV T1 } \\
\mathrm{cm}^{3}\end{array}$ & $\begin{array}{l}\text { htTKV } \\
\text { T0 }\end{array}$ & $\begin{array}{l}\text { eGFR decline } \\
\mathrm{mL} / \mathrm{min} / 1.7 \\
3 \mathrm{~m} 2 / \text { month }\end{array}$ \\
\hline 1 & 43 & 67 & 184.4 & 327.08 & 73 & 68 & 750 & 841 & 422 & -0.17 \\
\hline 2 & 51 & 122 & 189.6 & 304.94 & 62 & 58 & 629 & 602 & 602 & -0.44 \\
\hline 3 & 40 & 134 & 193.6 & 291.34 & 107 & 63 & 858.8 & 1045.7 & 536 & -0.56 \\
\hline 4 & 21 & 82 & 440.3 & 61 & 95 & 91 & 302.5 & 307 & 168 & -0.06 \\
\hline 5 & 48 & 82 & 323.6 & 422.25 & 47 & 61 & 2035 & 2380 & 1197 & -0.47 \\
\hline 6 & 48 & 54 & 201 & 389.47 & 40 & 45 & 1033 & 1260 & 1032 & -0.28 \\
\hline 7 & 34 & 73 & 104 & 265.32 & 71 & 53 & 559.7 & 615.4 & 342 & -0.39 \\
\hline 8 & 46 & 92 & 50 & 124.74 & 83 & 80 & 519.2 & 505.6 & 295 & -0.28 \\
\hline 9 & 51 & 66 & 244.3 & 1228 & 22 & 12 & 6360 & 9897 & 3438 & -0.29 \\
\hline 10 & 30 & 100 & 172 & 277.74 & 52 & 55 & 818.4 & 849 & 508 & -0.12 \\
\hline 11 & 33 & 87 & 141 & 298.67 & 56 & 53 & 839.6 & 923.5 & 486 & -0.10 \\
\hline 12 & 48 & 82 & 148 & 157.88 & 65 & 36 & 915 & 1821 & 495 & -0.56 \\
\hline 13 & 33 & 85 & 102 & 303.07 & 36 & 32 & 1517 & 1674 & 1517 & -0.14 \\
\hline 14 & 29 & 112 & 120 & 57.17 & 89 & 80 & 409 & 471 & 409 & -0.36 \\
\hline 15 & 30 & 90 & 236 & 450 & 40 & 46 & 1980 & 2682 & 1165 & -0.44 \\
\hline
\end{tabular}

Table1: Patient's characteristics of the study. Abbreviations: ADPKD: Autosomal Dominant Polycystic Kidney Disease; TFV, total fibrotic volume; TKV, total kidney volume; htTKV, Height-adjusted total kidney volume; $\mathrm{TPV} / \mathrm{TKV} \%$, ratio between the total kidney volume and total perfusion volume expressed as a percentage; PAC, plasma aldosterone concentration. 

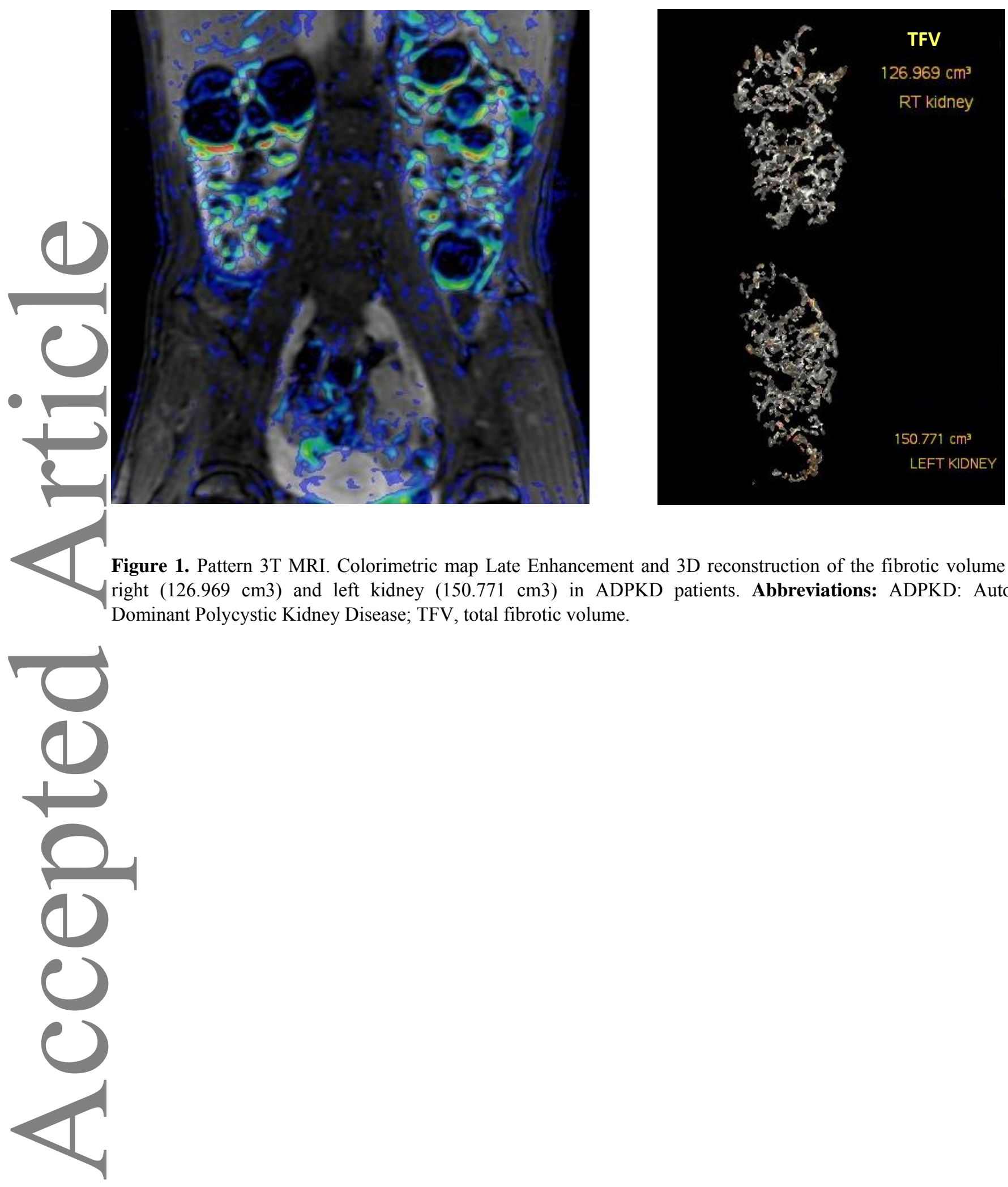

Figure 1. Pattern 3T MRI. Colorimetric map Late Enhancement and 3D reconstruction of the fibrotic volume of the right (126.969 $\mathrm{cm} 3)$ and left kidney (150.771 cm3) in ADPKD patients. Abbreviations: ADPKD: Autosomal Dominant Polycystic Kidney Disease; TFV, total fibrotic volume. 

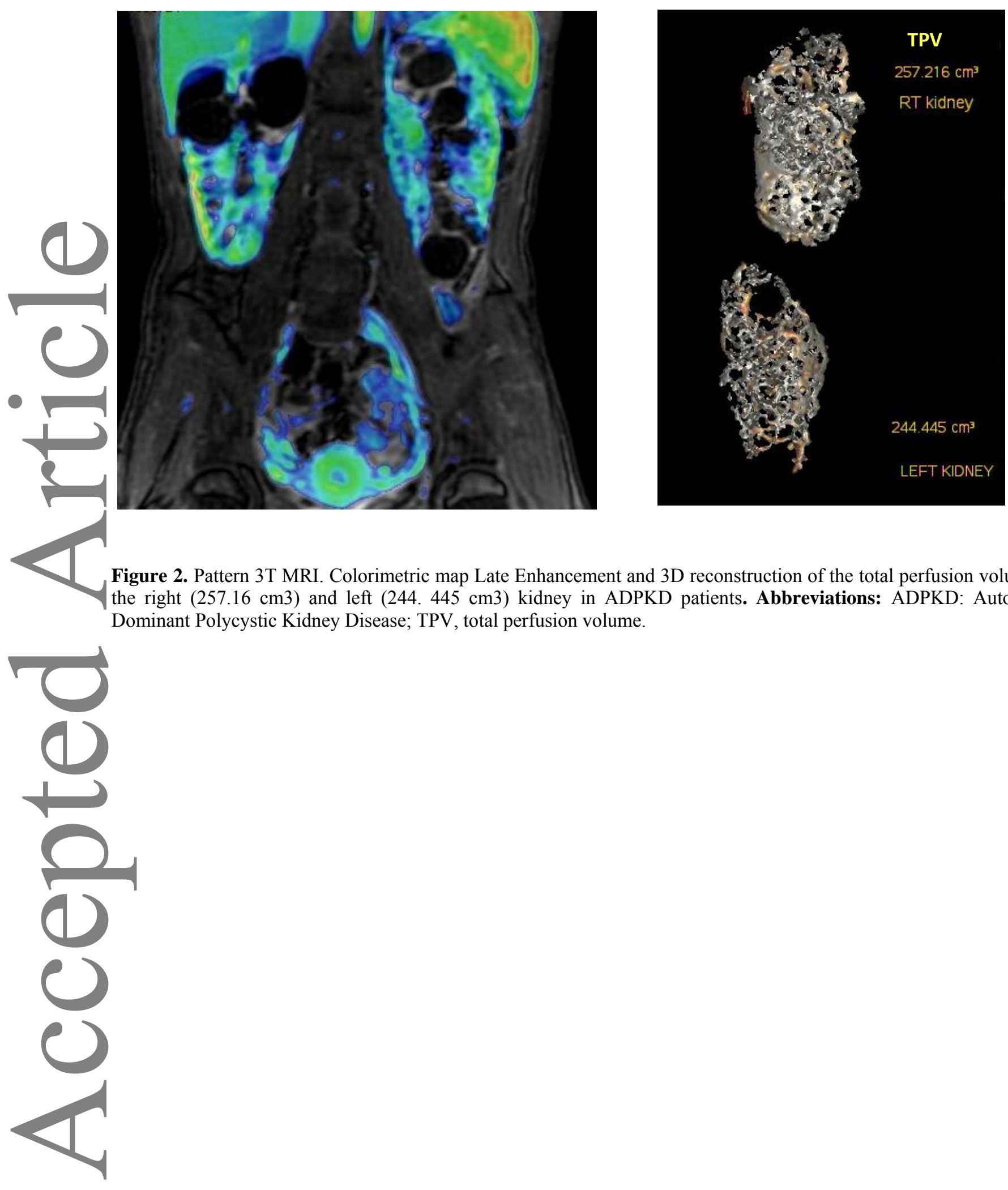

Figure 2. Pattern 3T MRI. Colorimetric map Late Enhancement and 3D reconstruction of the total perfusion volume of the right $(257.16 \mathrm{~cm} 3)$ and left $(244.445 \mathrm{~cm} 3)$ kidney in ADPKD patients. Abbreviations: ADPKD: Autosomal Dominant Polycystic Kidney Disease; TPV, total perfusion volume. 

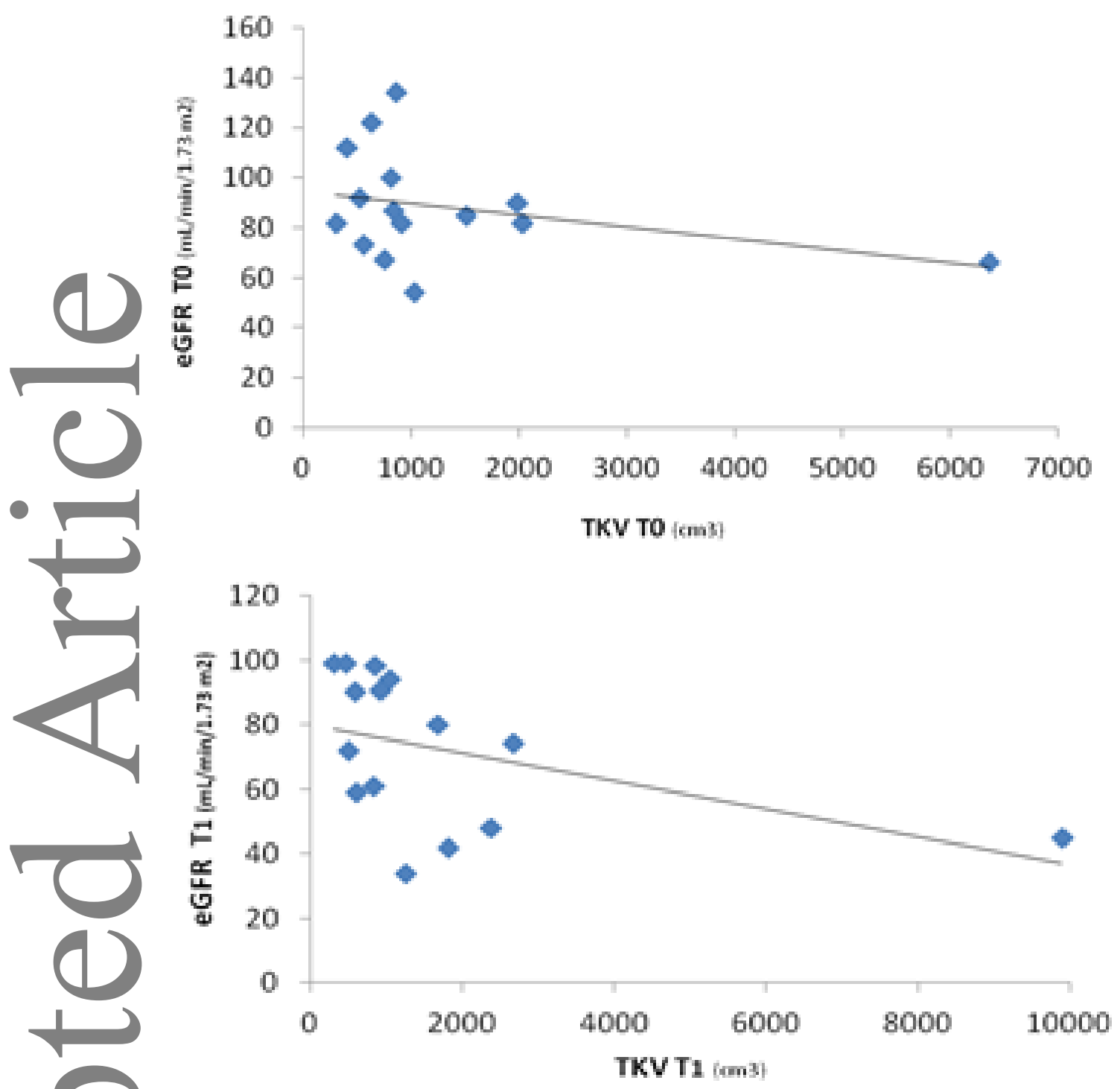

Figure 3. Correlation between the TKV and eGFR, at T0 $(r=-0.31, p=0.24)$ and at $T 1(r=-0.59, p=0.02)$ in ADPKD patients. Abbreviations: eGFR, estimated Glomerular Filtration rate; TKV, total kidney volume, ADPKD: Autosomal Dominant Polycystic Kidney Disease. 

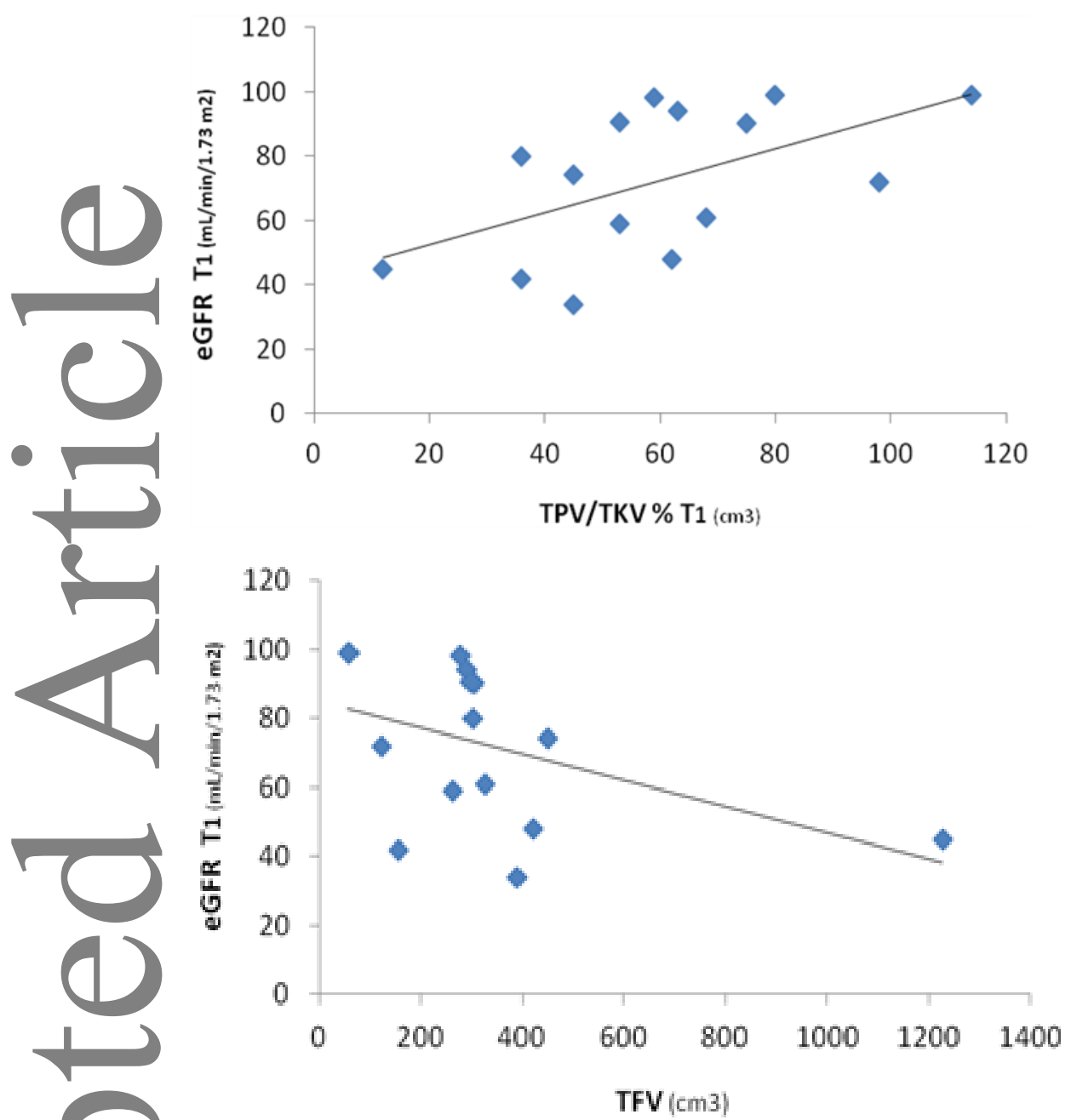

Figure 4: Correlation between the eGFR and TFV $(\mathrm{R}=-0.61, \mathrm{p}=004)$, and between eGFR and (TPV/TKV \%) $(\mathrm{r}=-$ $0.59, \mathrm{p}=0.02)$ at $\mathrm{T} 1$ in ADPKD patients. Abbreviations: TFV, total fibrotic volume; eGFR, estimated glomerular filtration rate; TPV / TKV\%, ratio between the total kidney volume and total perfusion volume expressed as a percentage; ADPKD: Autosomal Dominant Polycystic Kidney Disease. 